\title{
Uniaxial compression of single crystal and polycrystalline tantalum
}

\author{
G Whiteman ${ }^{1 *}$, S Case ${ }^{1}$, J C F Millett ${ }^{1}$, M J Cox ${ }^{1}$, P Avraam ${ }^{1}$, J P Dear ${ }^{2}$, A. Sancho ${ }^{2}$ and P A Hooper ${ }^{2}$ \\ ${ }^{1}$ AWE, Aldermaston, Reading, RG7 4PR, United Kingdom. \\ ${ }^{2}$ Department of Mechanical Engineering, Imperial College London, London, SW7 2AZ, United \\ Kingdom.
}

\section{ABSTRACT}

A series of compression experiments characterising the elastic-plastic response of single crystal and polycrystalline tantalum from quasi-static to intermediate strain-rates $\left(10^{-3}-10^{3} \mathrm{~s}^{-1}\right)$ over a range of temperatures $(233-438 \mathrm{~K})$ are reported in this paper. The single crystal experiments show significant differences in the response of the three principle crystal orientations of tantalum in terms of yield, work hardening and ultimate deformed shapes. Modelling is undertaken using a dislocation mechanics based crystal plasticity finite element model giving insight into the underlying microscopic processes that govern the macroscopic response. The simulations show the importance of the dislocation mobility relations and laws governing the evolution of the mobile dislocation density for capturing the correct behaviours. The inclusion of the twinning/anti-twinning asymmetry is found to influence [100] orientation most strongly, and is shown to be critical for matching the relative yield strengths. In general the simulations are able to adequately match experimental trends although some specific details such as exact strain hardening evolution are not reproduced suggesting a more complex hardening model is required. 3D finite element simulations approximating the tests are also undertaken and are able to predict the final deformed sample shapes well once the twinning/anti-twinning asymmetry is included (particularly for the [100] orientation). The polycrystalline data in both as-received and cold rolled conditions shows the initial yield strength is highest and work hardening rate is lowest for the cold-rolled material due to the increase in mobile dislocation density caused by the prior work. The general behavioural trends with temperature and strain-rate of the polycrystalline materials are reproduced in the single crystal data however the specific form of stress versus strain curves are significantly different. This is discussed in terms of the similar active slip systems in polycrystalline material to high symmetry single crystals but with the significant added effect of grain boundary interactions.

Keywords: Single crystal tantalum, crystal plasticity, polycrystalline tantalum, uniaxial stress

\footnotetext{
* Corresponding author: glenn.whiteman@awe.co.uk
} 


\section{INTRODUCTION}

The plasticity of polycrystalline tantalum has been extensively studied in previous decades over a large range of strain-rates and temperatures ${ }^{1-6}$. Tantalum, along with other body centred cubic (BCC) metals, exhibits a number of distinctive properties that distinguish it from, for example, face centred cubic (FCC) metals, such as: the dominance of screw dislocations in defining dislocationmediated plasticity ${ }^{7}$, strong strain-rate and temperature dependence of the yield stress that can be attributed to thermal activation over a large Peierls barrier ${ }^{8}$, violation of the Schmid law ${ }^{9}$ that predicts slip occurs on a slip system when the resolved shear stress reaches a critical value (which is a material constant/property) ${ }^{10,11}$, and ambiguity in the operative slip systems ${ }^{12}$. Although a plethora of experiments on polycrystalline tantalum have been performed ${ }^{8,13-20}$, interaction between the constitutive single crystal grains can complicate interpretation of the underlying fundamental plasticity mechanisms. Recent studies ${ }^{21}$ have shown that single crystal effects play a key role in influencing the macroscopic response of polycrystals, and so an accurate description of these effects is sought through experiments on single crystal materials.

Published data on single crystal tantalum in low strain-rate compression and tension experiments at various temperatures 10, 11, 22-25 have revealed non-Schmid effects, tensioncompression asymmetry, strong directionality of the yield stress, and sensitivity to impurity content. Many of the non-Schmid effects are explained, at least qualitatively, by assuming that resistance to glide on $\{112\}$ planes is lower in the twinning sense than the anti-twin sense ${ }^{26}$. Microstructural analyses on single crystals deformed along [110] ${ }^{23}$, revealed localised rotations within the crystal due to plastic slip, but no evidence of other dislocation mechanisms or twinning. At higher strainrates (of the order $10^{3} \mathrm{~s}^{-1}$ ) split Hopkinson pressure bar (SHPB) techniques have been used to load $[100]$ and $[110]$ directions ${ }^{27}$, and $[\overline{149}]$ and $[\overline{144}]$ directions at a range of temperatures ${ }^{28}$. A strong dependence of yield stress and strain hardening rate on crystal direction was seen. Single crystals deformed along [110], under uniaxial stress conditions, at both quasi-static ${ }^{23}$ and SHPB ${ }^{27}$ strainrates show essentially plane strain that endures to large strains, with initially cylindrical samples taking on an ovoid cross-section. At higher rates still $\left(\geq 10^{5} \mathrm{~s}^{-1}\right)$ ramp loading ${ }^{29,30}$ plate impact ${ }^{31-33}$ and laser driven ${ }^{34}$ experiments on some orientations of single crystal tantalum have all been reported noting various orientation dependencies on the measured response and active deformation mechanisms. 
Crystal plasticity (CP) finite element models, like those described by Bronkhorst et al. ${ }^{35}$, are being developed to predict material behaviour across a range of loading strain-rates and temperatures. These models give the possibility of exploring microscopic processes and the deformation of anisotropic heterogeneous crystalline materials by including descriptions of elasticplastic deformation mechanisms in single crystals. Single crystal experiments offer a stringent tests of these CP models and a more direct link to microscopic deformation. In order to model the elasticplastic response of $\mathrm{BCC}$ tantalum over a large range of strain-rates requires accurate account of thermal activation processes, phonon drag processes for stresses that exceed the Peierls stress and dislocation density evolution. Account of the mobile dislocation density evolution is key in predicting many observations in shock deformation, such as elastic precursor decay ${ }^{36,37}$ and the peaks seen in precursor waves ${ }^{38}$, while hardening behaviour, that depends on dislocation-dislocation interactions, is intimately linked with evolution of the dislocation density. Together with inclusion of the aforementioned non-Schmid effects, accurate modelling in these regimes is challenging.

The aim of the current study is to experimentally characterise the low and intermediate rate elastic-plastic compression response of single crystal tantalum in the three principle orientations ([100], [110] and [111]) and polycrystalline tantalum in both as-received and cold rolled conditions. To this purpose, uniaxial stress compression experiments have been performed at strain-rates of $10^{-3} \mathrm{~s}^{-1}, 10^{-1} \mathrm{~s}^{-1}$, and $10^{3} \mathrm{~s}^{-1}$, and at temperatures between $233 \mathrm{~K}$ and $438 \mathrm{~K}$. These experiments are part of an ongoing programme to characterise the response of tantalum across a range of strainrates $^{20,33,39-44}$ to provide benchmark data for CP finite element model development, gaining insight into the underlying microscopic processes that govern the macroscopic response. The single CP model presented here has been developed to be applicable over a large range of strain-rates from quasi-static and intermediate rates $\left(\leq 10^{4} \mathrm{~s}^{-1}\right)$, shown in this paper, up to shock loading $\left(\geq 10^{5} \mathrm{~s}^{-1}\right)$.

\section{MATERIALS AND EXPERIMENTS}

\subsection{Materials}

Three bars of approximately $14 \mathrm{~mm}$ diameter single crystal tantalum were obtained from Metal Crystals and Oxides Ltd, Cambridge, UK. The bars were grown with axes parallel to the [100], [110] and [111] crystallographic directions. Reported mis-orientation was less than $0.5^{\circ}$ and the purity was $99.99 \%$ minimum. Transmission electron microscopy (TEM) bright field images of the undeformed 
materials are presented in Figure 1. All three orientations show low levels of dislocation density measured to be $\sim 6 \times 10^{12} \mathrm{~m}^{-2}$ in each sample. The TEM also confirmed the single crystal nature of the materials, showing no evidence of low angle grain boundaries. Measured interstitial contents showed oxygen to be the primary impurity, present in the range $130-180 \mathrm{ppm}$.

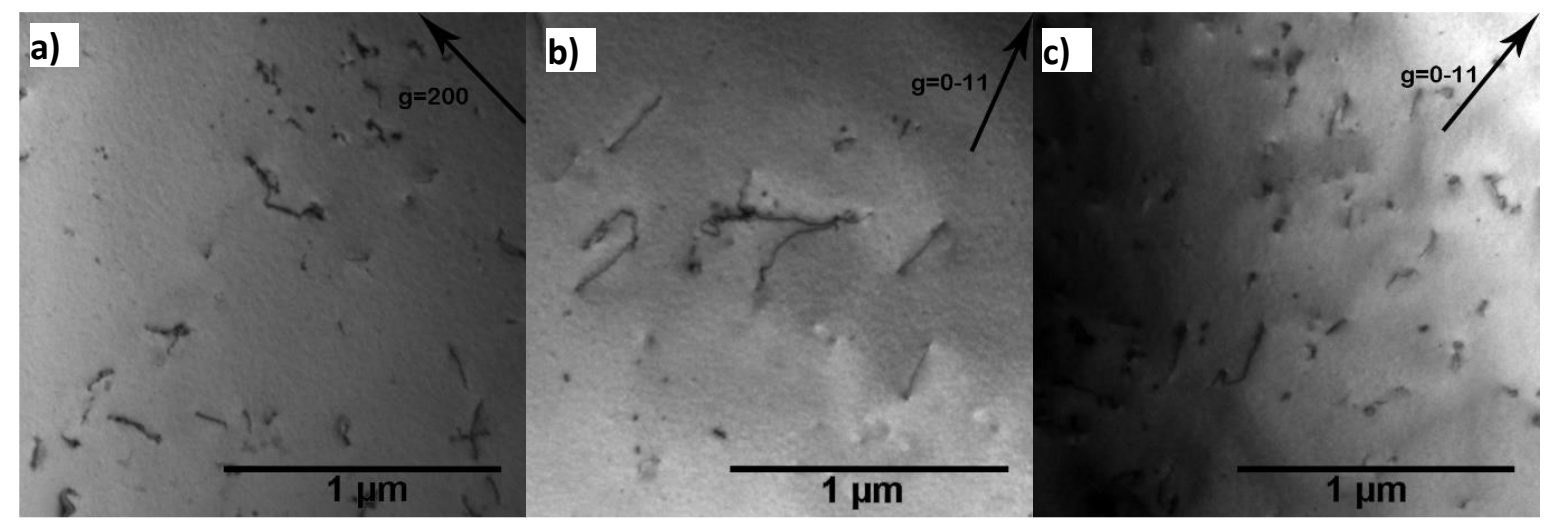

Figure 1: TEM images of the initial single crystal tantalum samples: (a) [100], (b) [110], (c) [111].

The polycrystalline tantalum used for this research is of the same batch as used in previously reported shock experiments ${ }^{20}$. In its as-received condition it displays a texture preference for the $<001>$ direction, a relatively low dislocation density with the dislocations arranged in a loose cell structure. The same material was sequentially cold-rolled in one direction to a reduction in thickness of $50 \%$ putting in an equivalent strain of 0.8 . Electron back scatter diffraction revealed that the coldrolled material showed little change in texture aside from a degree of grain rotation. The dislocation density appeared to have increased by only a small amount, although dislocations arranged themselves into a more clearly defined cell structure.

\subsection{Experiments}

Quasi-static compression and split Hopkinson pressure bar (SHPB) experiments were undertaken in the Mechanical Engineering Department, Imperial College London. Approximately 90 uniaxial stress compression experiments were performed in total using the three orientations of single crystal tantalum and polycrystalline tantalum in as-received and cold-rolled conditions. Cylindrical samples of approximately $4.5 \mathrm{~mm}$ length and $4.5 \mathrm{~mm}$ diameter were compressed in three strain-rate regimes, $\sim 10^{-3}, 10^{-1}$ and $10^{3} \mathrm{~s}^{-1}$ and three temperatures, $233 \mathrm{~K}, 293 \mathrm{~K}$ and $438 \mathrm{~K}$ (or $415 \mathrm{~K}$ for the 
highest rate SHPB experiments). The low and intermediate strain-rate experiments were carried out using an Instron 5584 load frame machine with an integral furnace/cooling chamber. Samples were taken to large total true strains ( $70 \%)$. For the higher strain-rate experiments, the samples were heated/cooled in a temperature chamber for $~ 10$ minutes before being transferred in a PTFE insulating holder to the SHPB. The SHPB was modified in order to bring the bars into contact with the sample and fire in a short period of time, $\sim 230 \mathrm{~ms}$ from contact until the compression pulse entered the input bar. This ensured minimal heating/cooling of the samples. The sample temperature stated is based on a number of thermocouple test runs such that the final temperature is estimated to be accurate to $\pm 2{ }^{\circ} \mathrm{C}$. A momentum trap was also used on the SHPB in these experiments to ensure that the samples were only struck once. For the SHPB samples this led to total strains of between 10-20\%. A number of the compressed single crystal samples from a selection of rates and temperatures were additionally examined using $\mathrm{X}$-ray tomography to recreate a full 3D representations of the deformed shapes for comparison with the crystal plasticity model predictions.

\section{RESULTS AND DISCUSSION}

\subsection{Single Crystals}

Typical quasi-static true stress versus true strain data is shown in Figure 2(a) for the three single crystal orientations at $233 \mathrm{~K}$. Significant differences in response between the materials are apparent. For example, the yield strength is highest for the [111] orientation and lowest for the [110] orientation, suggesting variation in active slip systems. Work hardening, up to 10\% strain, is largest for the [100] orientation and smallest for [110]. Also apparent for the [111] orientation is the presence of an upper and lower yield point, seen at 1-2\% strain, and a large dip and reload behaviour in true stress above $\sim 10 \%$ strain. The occurrence of an upper and lower yield point indicates that the stress required to maintain plastic flow in the [111] crystal immediately after yielding is lower than that required to start it. The dip and reload behaviour is the result of a significant shearing action which can be observed in the recovered [111] orientation samples which had a complex collapsed shape. None of the post-test recovered samples for any orientation were perfectly cylindrical as indicated in Figure 2(b) with typical X-ray tomographic scans of low-rate tested samples. It was observed that recovered [110] crystals were oval in cross-section, i.e. they 
exhibited approximately "plane strain" deformation, with plastic strain essentially negligible along one direction and finite along the other two, in agreement with previous quasi-static and SHPB uniaxial stress experiments ${ }^{23,}{ }^{27}$. In general the [100] crystals exhibited a square-like cross-section with 4-fold mirror symmetry although were more elongated in the high temperature tests. This differs from previously reported data 27 where we infer a that compressed [100] samples remained circular. Insight into the origins of all these shapes are discussed later. Overall it appears that conditions of uniaxial stress are maintained in these deformations, with the exception of the [111] orientation samples past $\sim 10 \%$ strain for the low-rate experiments.

a)

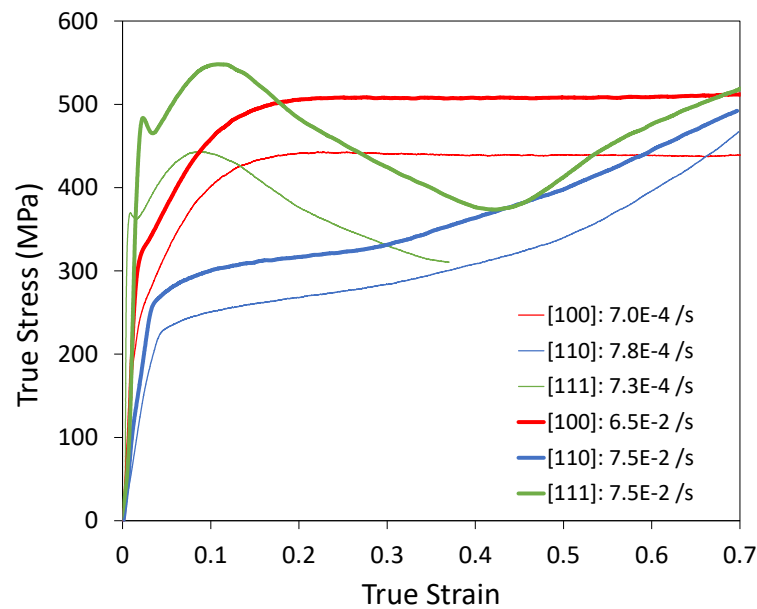

b)

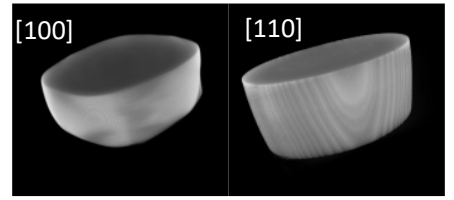

[111]

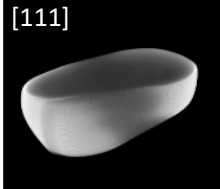

Figure 2: a) True stress - true strain data for low rate compression tests carried out at $233 \mathrm{~K}$ on the three orientations of single crystal tantalum, shown up to true strains of $\sim 70 \%$. b) X-ray tomographic scans showing 3D surface shape of the three orientations of single crystal as deformed at room temperature at $\sim 7 \times 10^{-4} \mathrm{~s}^{-1}$.

${ }^{a}$ There is a discrepancy in the paper by Rittel et al. ${ }^{27}$ as the final shapes noted in the text and shown in figure 4 do not agree. We have inferred that it was their [100] crystals which remained circular due to the agreement with the oval shapes of the [110] crystals. 
a)

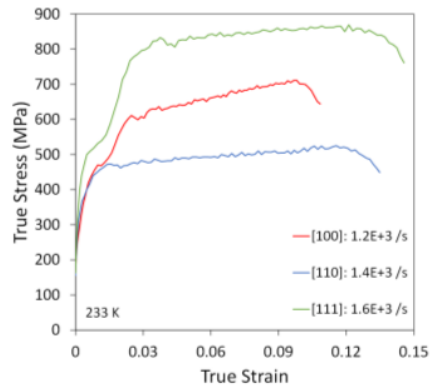

b)

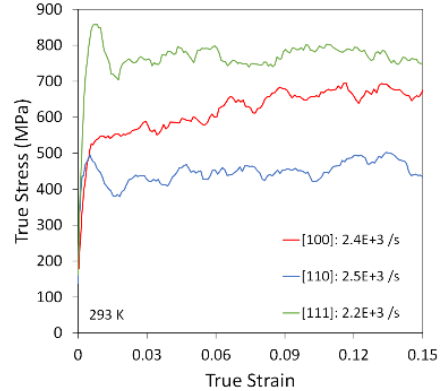

c)

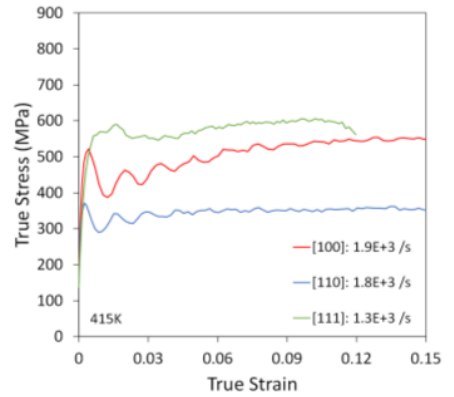

Figure 3: Typical true stress - true strain data for SHPB experiments on single crystal Ta at a) low temperature (233K), b) room temperature (293K) and c) high temperature (415K). Note that the strain-rates stated are the mean rates measured from yield to 15\%.

The initial temperature effect on the single crystal samples are shown for the higher rate SHPB data in Figure 3. The stress versus strain data shows the same consistent ordering of yield stress at all temperatures between the crystals as observed at lower rates, with [111] being the strongest followed by [100] and [110]. These relative yield stresses are consistent with other experimental studies ${ }^{24,27}$, although not with the work of Byron et al. ${ }^{10}$ where the yield stress of [110] appears larger than [100]. In general the [111] and [110] orientations exhibit a similar work hardening after yield while the [100] hardens more significantly at all rates. All materials exhibit a significant degree of both temperature and strain-rate sensitivity.

The flow stress determined at $10 \%$ true strain for the data is presented in Figure 4 as a function of initial sample temperature (Figure $4(a)$ ) and average strain-rate for the room temperature experiments (Figure $4($ b)). Here we have also taken the opportunity to compare to previously reported data on [100] and [110] single crystal tantalum ${ }^{27}$. 
a)

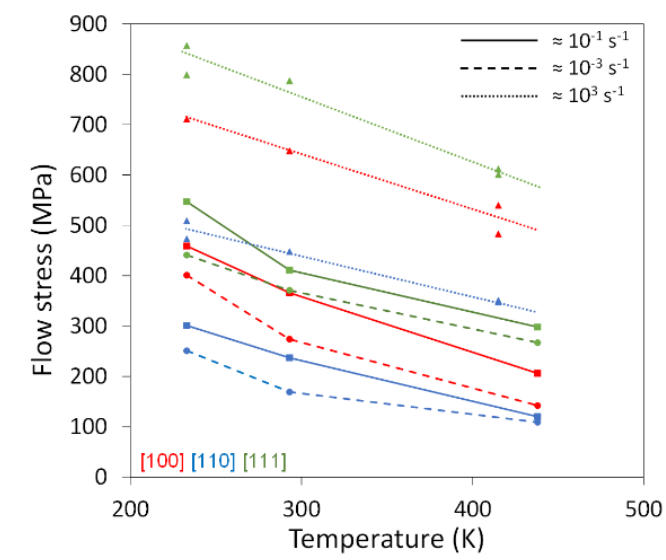

b)

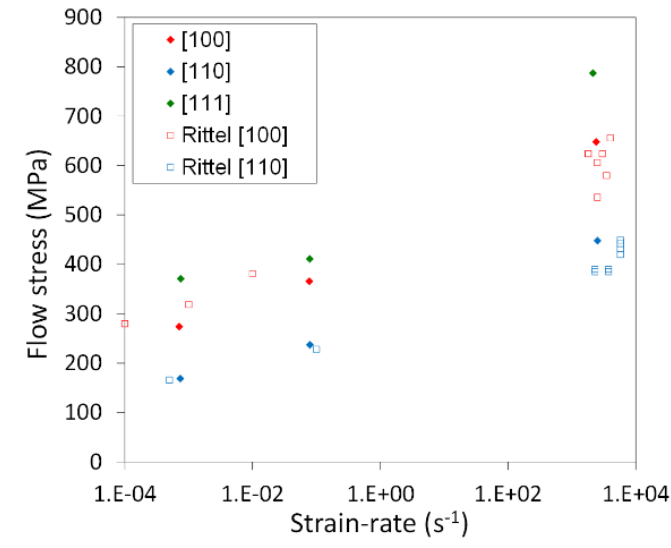

Figure 4: Flow stress determined at $10 \%$ true strain for single crystal tantalum samples plotted versus a) initial sample temperature and b) average test strain-rate at room temperature compared to previously reported data ${ }^{27}$.

The temperature dependence of the materials is shown clearly in Figure 4(a), with the ordering of flow strength consistent across the range tested. The flow strength for all three orientations decay in a similar manner with increasing temperature and for all strain-rates. The rate dependence is shown more clearly in Figure 4(b) indicating the increase of flow strength with strain-rate. The [111] data displays a slightly larger rate-dependence than the other orientations. The figure also shows the general agreement with the [100] and [110] data presented by Rittel et al. ${ }^{27}$

It is noted that research practicalities in this case did not allow a large number of repeated experiments to be undertaken as would normally be desired. One nominal repeat of each high and low temperature test at high strain-rates was undertaken for all three orientations, with the data shown in Figure 4(a). In general, overall stress-strain behaviour and final sample shapes were consistent but with small variations in flow stress similar in magnitude to those of Rittel et al. ${ }^{27}$ shown in Figure 4(b). These experiments have been modelled using a dislocation mechanics based CP finite element model. The model is based on those presented by Bronkhorst et al. ${ }^{35,45,46}$ but also includes a physically based model for slip kinetics to provide a description of the evolution of the dislocation sub-structure, similar to that presented in other papers ${ }^{1,47,48}$. The model, developed to be applicable over a large range of strain-rates from quasi-static $\left(\leq 10^{-3} \mathrm{~s}^{-1}\right)$ to shocks $\left(\geq 10^{5} \mathrm{~s}^{-1}\right)$, describes dislocation velocity by thermal activation and dislocation drag processes (fitted to molecular dynamics data) and includes evolution of the mobile and immobile dislocation densities (fitted to shock data). Twinning in BCC metals is more likely at low temperatures and high strainrates ${ }^{49}$, however only dislocation-mediated plasticity is currently considered in the model. It is assumed that the current simulations represent slip planes in an average sense, and so slip on $\{110\}$ 
and $\{112\}$ planes in $<111>$ directions is considered. A non-Schmid effect resulting in a twin/anti-twin (T/AT) asymmetry has been introduced by assuming that resistance on $\{112\}$ planes is larger in the anti-twin sense. The full details of the model will be presented in a future paper.

Simulations of the three crystal orientations were first undertaken across the range of strain-rates and temperatures applied in these experiments using a single finite element cell to explore the stress-strain response. Example simulation and experiment data for the room temperature SHPB experiments are shown in Figure 5. Suppression of the $\{112\} A T$ slip systems was key to predicting relative initial yield stress magnitudes in agreement with the experiments for the three directions. Without the alteration, [100] and [110] initial yield stress predictions were of similar magnitude to each other. With the alteration, only the [100] results were affected, seeing an increase in yield stress. Suppression of $\{112\} A T$ systems means that in the [100] simulation the $\{110\}\langle 111\rangle$ systems that are less favourably oriented must be employed to accommodate the imposed plastic strain, and thus the yield stress is larger. For deformation in the [110] and [111] directions, $\{112\}$ AT systems are not significantly active using a Schmid model, and so their suppression has negligible effect on the stress-strain response. The general experimental trends (initial yield stress, hardening rates, and the relative flow stress magnitudes between the three directions) were described fairly well by the model, although specific details are not. For example, exact details of the strain hardening rates are not reproduced. In the data shown in Figure 5 it can be seen that the [100] orientation displays a larger hardening rate (up to strains of 0.1). The [100] orientation has more available slip systems and the hardening behaviour, which depends on dislocation-dislocation interactions, is intimately linked with evolution of the dislocation density. Together with inclusion of the aforementioned nonSchmid effects, accurate modelling in these regimes is challenging. More complex hardening models (e.g. Stainier et al. ${ }^{50}$ ) could be employed in future work to address this shortcoming. It is also noted that when modelling was applied to the low and high temperature experiments the comparison to experiment was similar in all cases except for the [111] orientation at high temperatures. At this condition the model produced flow stresses significantly lower ( $100 \mathrm{MPa})$ than the experimental results. This suggests that a significant effect is unaccounted for in the applied model, for example slip on $\{123\}$ planes which is more likely at higher temperatures [51]. 


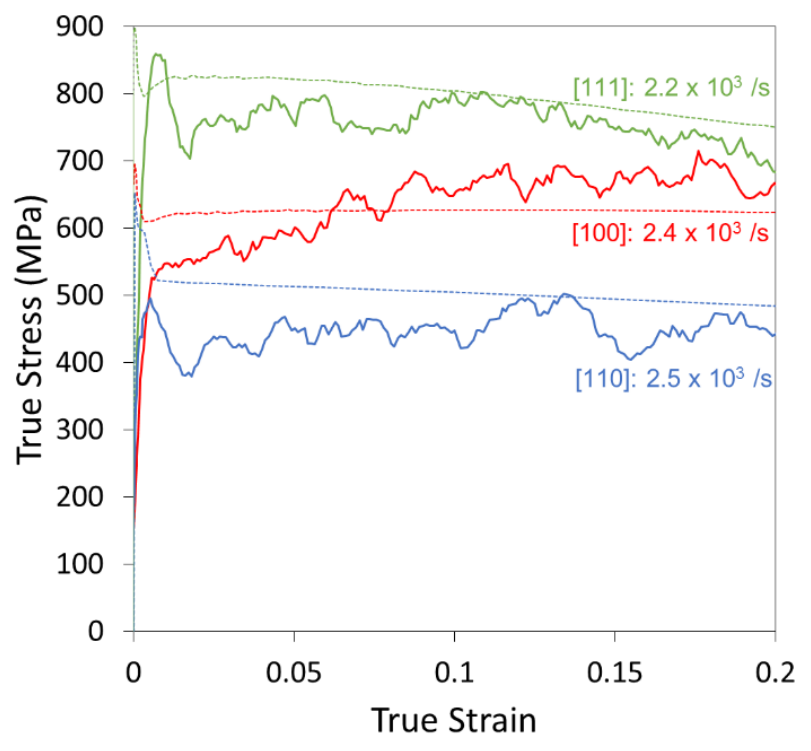

Figure 5: Simulations of SHPB uniaxial stress tests using a single finite element. Experiment: solid lines, simulation: dashed lines.

In general the strain state for single crystals is not uniquely defined by the standard measurements made in uniaxial stress compression tests. As such the stress-strain values must be used carefully in any assessment. The intention for this research is the comparison of experiment and modelling to develop and validate crystal plasticity models. As such, in addition to the single cell simulations, 3D finite element simulations approximating the SHPB tests were also performed. Here the models are able to directly compare to, and give insight into, the experimental deformed sample shapes. Figure 6 compares the $x$-ray tomographic scans of the post experiment shapes with simulated shapes at approximately equivalent axial strains. 


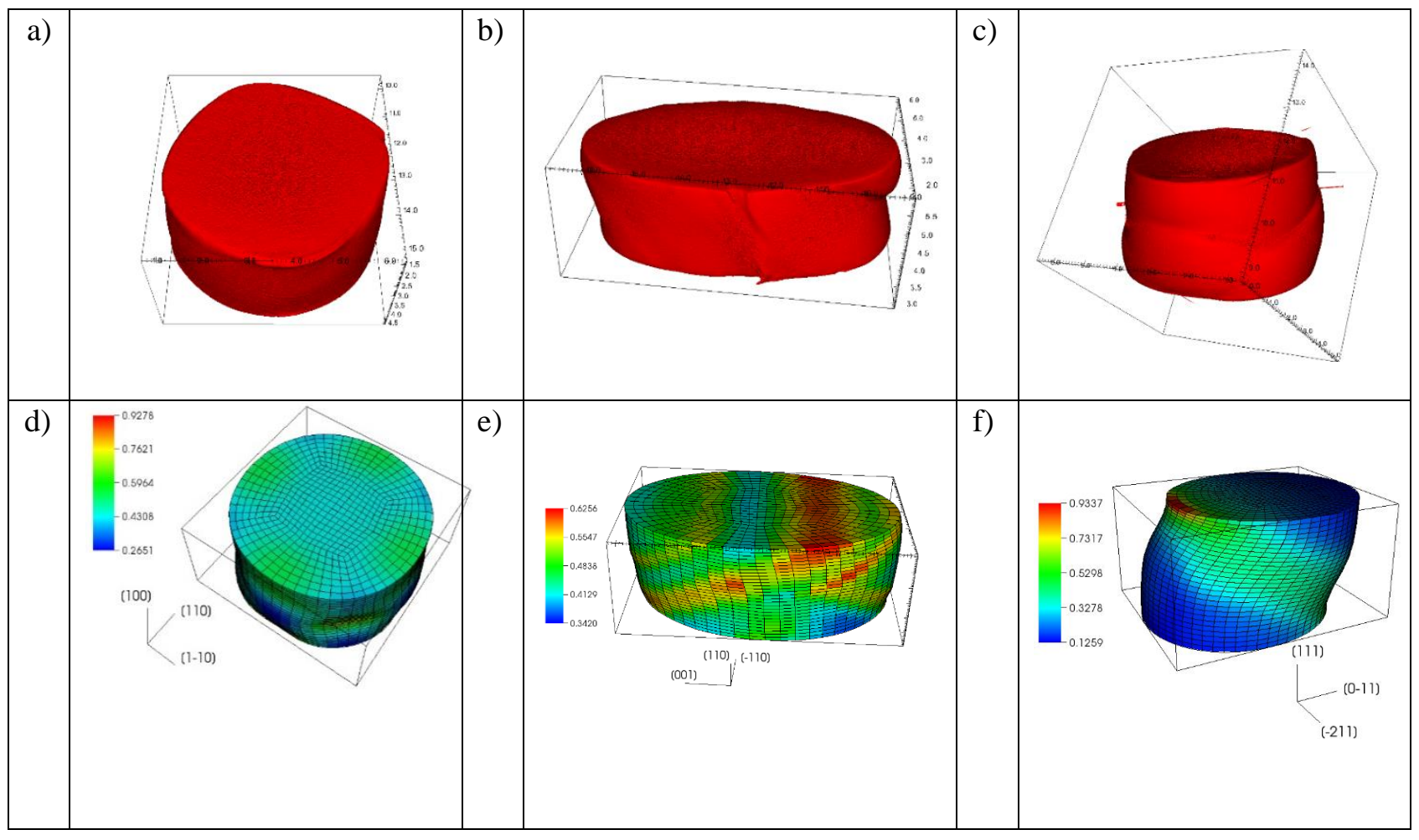

Figure 6: Comparison of 3D tomographic scans of SHPB exeriments and simulations. Experiments: (a) [100], (b) [110], (c) [111]; simulations: (d) [100], (e) [110], (f) [111]. Length units are in mm. Colour scale in simulations signifies equivalent plastic strain contours.

The final sample shape for the [100] SHPB simulation Figure 6(d) is seen to be in fairly good qualitative agreement with the experiment, showing a square-like cross-section with 4-fold mirror symmetry. Suppression of the (112)AT systems was key in achieving this agreement; a conventional Schmid model produced deformed specimens having a circular cross-section. Deformation features on the sides of the specimen are present in the simulations that are not seen in the experiment. This difference is not understood, but could be related to transient effects arising from the approximate boundary conditions applied here.

For the [110] SHPB simulations, better agreement with the experimental sample shape was obtained by offsetting the compression direction by $0.5^{\circ}$ from ideal (i.e. within the crystal mis-orientation accuracy). The simulated final sample shape, Figure 6(e), is seen to be in good qualitative agreement with the experiment Figure 6(b). The simulation and experiment exhibit approximately "plane strain" deformation, with plastic strain essentially negligible along [110] and finite along [110] (the compression axis) and [001]. The [110] single cell simulation revealed the active systems to be: 


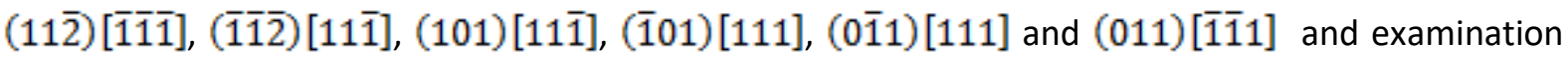
of these systems shows they are not expected to contribute plastic strain in the [110] direction. The experimental specimen shows localised deformation features that the simulations are not expected to reproduce, although the onset of features of this type is evident in the simulations in Figure 6(e).

Final sample shapes in all of the [111] experiments taken to large strains consistently showed a heavily sheared shape. The onset of shear in this high-rate, lower strain example can be seen in the scanned sample in Figure 6(c). Simulations of deformation exactly along [111] did not yield sample shapes in agreement with experiment. Offsetting the compression direction by a fraction of a degree from ideal yielded better agreement, as shown in Figure $6(f)$ for a sample offset by $0.1^{\circ}$. The simulated sample sides do not remain parallel, and the sample undergoes shear, similar to the deformation that occurred in the experiments. Only three slip systems are significantly activated in

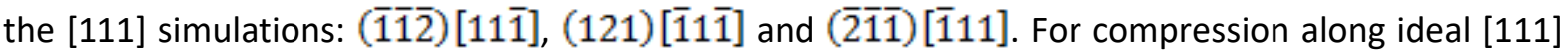
(achievable in simulations), the systems experience identical resolved shear stresses, are activated to an equal degree, and their symmetry means the sample deforms uniformly, with the sides remaining parallel. When compression occurs along a direction slightly offset from [111] (as in experiments), the systems experience different resolved shear stresses, are activated to different degrees, and the sample does not deform uniformly.

\subsection{Polycrystalline material}

Typical quasi-static true stress versus true strain data are shown in Figure 7 comparing the behaviour variation with temperature of as-received and cold rolled polycrystalline tantalum at intermediate and high strain-rates. 
a)

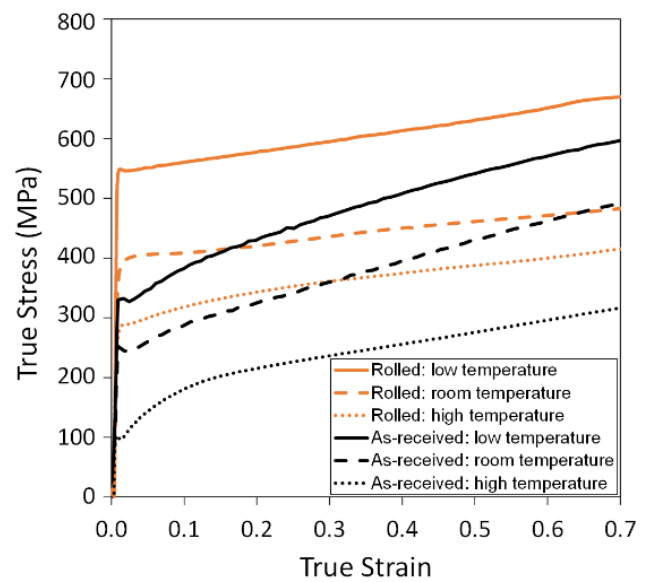

b)

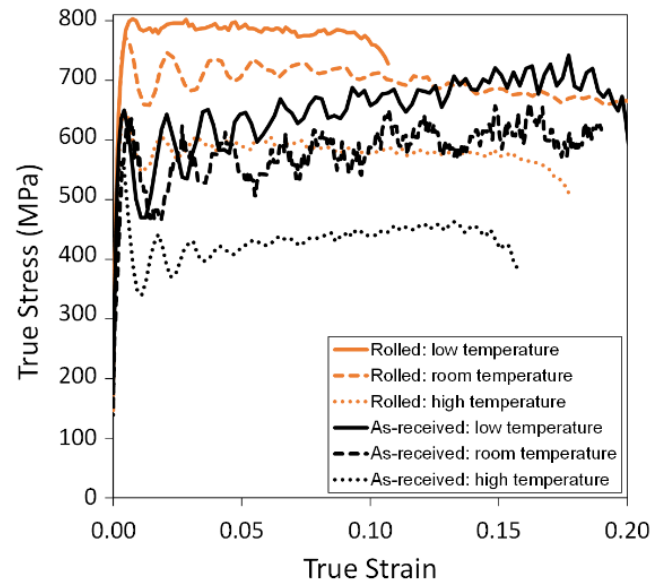

Figure 7: Typical true stress - true strain data for polycrystalline as-received and cold rolled tantalum obtained for a) intermediate strain-rates $\sim 10^{-1} \mathrm{~s}^{-1}$ and b) high strain-rate SHPB experiments, $\sim 10^{3} \mathrm{~s}^{-1}$. LT = low temperature (233K), RT = room temperature $(293 \mathrm{~K})$ and $\mathrm{HT}=$ high temperature (438K or $415 \mathrm{~K}$ for SHPB).

It can be seen that both yield and work hardening characteristics differ between the as-received and cold-rolled materials similar to the behaviour observed by Chen and Gray ${ }^{8}$ and Florando et al. ${ }^{15}$ For example, the initial yield strength is highest and work hardening rate is lowest for the cold-rolled material due to the increase in mobile dislocation density caused by the prior work. In the asreceived material a more gradual parabolic strain-hardening is observed. Also apparent in the low and intermediate rate data for the as-received material was the presence of an upper and lower yield point, as seen in Figure 7(a) for the intermediate rate data. In tantalum pinning of dislocations by interstitial solutes (such as oxygen atoms) can lead to this feature. It was confirmed that the effect of increasing temperature on this feature was to reduce the size of the difference between upper and lower yield stress but to a lesser degree than the reduction in overall yield strength. After cold rolling, with the availability of more mobile dislocations due to both new line length and the removal of dislocations from the pinning interstitials, the upper and lower yield behaviour is removed and a sharp transition from elastic to plastic is observed.

The flow stress at $10 \%$ true strain for the data is presented in Figure 8 as a function of initial sample temperature (Figure 8(a)) and average strain-rate for the room temperature experiments (Figure $8(b))$. There appears to be very little strain-rate dependence across the range of temperatures investigated for either material. Florando et al. ${ }^{15}$ observed a decrease with temperature of the difference between flow stress at different rates. In our case the minimum temperature of $233 \mathrm{~K}$ is relatively high (compared to $77 \mathrm{~K}$ ) meaning that it is likely too high to limit the thermally activated 
kink-pair mechanism thought to be responsible for the strain-rate dependence observed in that research.

a)

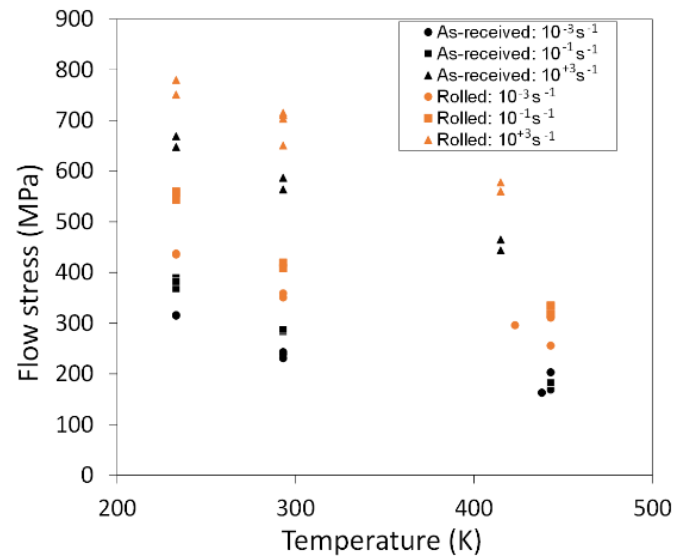

b)

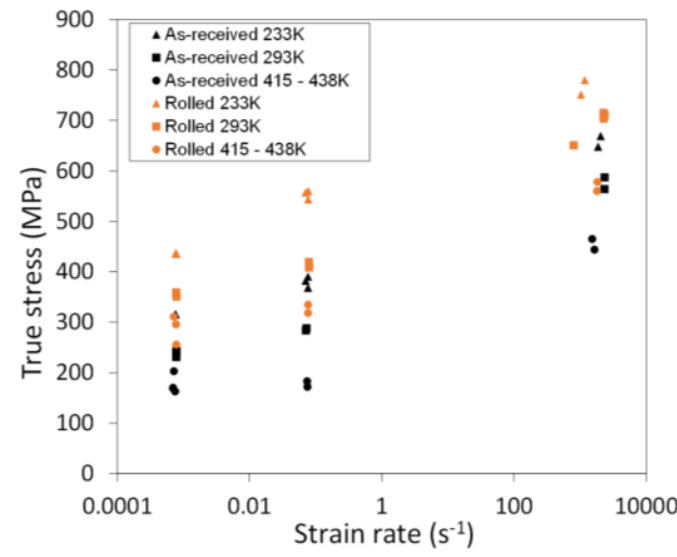

Figure 8: Flow stress determined at $10 \%$ true strain for polycrystalline tantalum samples plotted versus a) initial sample temperature for the three strain-rates and b) average test strain-rate at the three temperatures.

The general trends of flow stress with temperature and strain-rate for the polycrystalline materials are unsurprisingly similar to the single crystal materials. Overall the flow stress at 0.1 strain of the asreceived polycrystalline material appears nominally most similar to the [100] orientation data but is consistently 30-80 MPa lower. The shape of the stress strain curves are however notably different, with the [100] data being significantly more parabolic (up to 10\% strain) and crossing the polycrystalline curve at increasing strains with decreasing temperature, as shown in Figure 9. This suggests that while it's possible that slip systems in [100] oriented crystallites dominate the mechanical response of polycrystalline tantalum as suggested by Rittel et al. ${ }^{27}$, the specific slip and dislocation interactions are not simply relatable. For example, grain boundary interactions may tend to activate multiple slip systems making polycrystalline yield behaviour most like a higher symmetry orientation single crystal but dislocation interactions with the grain boundary make later time hardening behaviour quite dissimilar. 


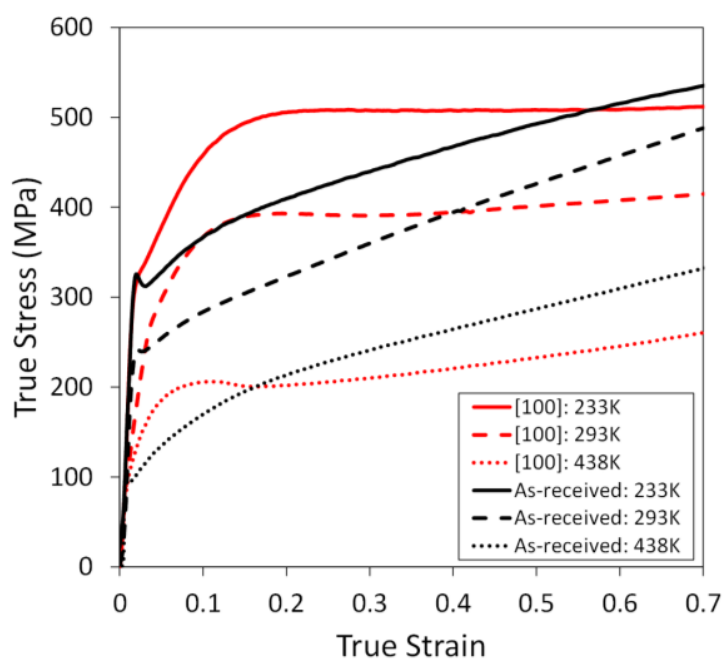

Figure 9: True stress - true strain data for polycrystalline as-received and [100] single crystal tantalum obtained at $\sim 10^{-1} \mathrm{~s}^{-1}$ at three initial temperatures. 


\section{CONCLUSIONS}

A series of uniaxial stress compression experiments have been undertaken on single crystal and polycrystalline tantalum. These experiments are part of an ongoing programme to characterise the elastic-plastic compression response of tantalum across a range of strain-rates ${ }^{20,33,39-44}$ to provide benchmark data for CP model development.

The data presented in this paper shows the significant differences in the response of the three principle crystal orientations of tantalum in terms of yield, work hardening and ultimate deformed shapes. The simulations presented highlight the importance of the dislocation mobility relations and the laws governing the evolution of the mobile dislocation density for capturing the correct behaviours. The inclusion of the twinning/anti-twinning asymmetry was found to influence [100] oriented compression most strongly, and was shown to be critical for matching the relative yield strengths in these experiments. In general we were able to adequately match experimental trends although some specific details such as exact details of strain hardening evolution were not reproduced suggesting a more complex hardening model could be employed in the future. 3D finite element simulations approximating the SHPB tests predicted the final deformed sample shapes well once the twinning/anti-twinning asymmetry was included (particularly for the [100] orientation) and small offsets of compression axis were included for [110] and [111] orientations. The model also describes the expected active slip systems in these experiments.

The polycrystalline data in both as-received and cold rolled conditions confirms the trends observed by other researchers ${ }^{8,15}$. The initial yield strength is highest and work hardening rate is lowest for the cold-rolled material which is consistent with a higher mobile dislocation density caused by the prior work. The difference between the upper and lower yield point in the as-received material reduced in size with an increase in temperature and was removed entirely by cold rolling as dislocations are removed from the pinning interstitials. The difference of flow stress at different strain-rates remains approximately constant for both polycrystalline materials as the initial temperature changes although the minimum temperature of $233 \mathrm{~K}$ used in this work is still likely to be too high to limit the thermally activated kink-pair mechanism. The general behavioural trends with temperature and strain-rate of the polycrystalline materials are reproduced in the single crystal data and the flow stress at 0.1 strain of as-received polycrystalline material appears nominally most similar to the [100] orientation. This suggests that polycrystalline materials, where the effect of grain 
boundary interactions will tend to activate multiple slip systems, will generally behave in a way most similar to a higher symmetry orientation single crystal like the [100]. However, given that the shape of the stress strain curves are significantly different, with the [100] data being more parabolic than the as-received polycrystalline tantalum, the dislocation interactions post yield are not as simply related.

The ultimate goal of this research is the ability to successfully match materials behaviour with a single model over a wide range of plastic strain-rates. While this is a significant challenge, the CP model performs well in this respect for single crystal tantalum both in these experiments and also against shock data (which will be the subject of a subsequent publication). Single crystal effects play a key role in influencing the macroscopic response of polycrystals and the longer term goal for this single crystal model is its incorporation into predictive polycrystal simulations using the known crystallographic structure and texture of the materials shown here. Further work will however be needed to develop the single crystal capability to achieve all these goals, to gain insight in and successfully predict the underlying microscopic processes that govern the macroscopic response of materials.

\section{ACKNOWLEDGEMENTS}

We would like to thank Dr. A. Worley formerly of Imperial College London for his assistance in undertaking the experiments presented here. We also acknowledge Prof. I. Jones, Dr. Y-L Chiu and Dr. B. Pang from the School of Metallurgy and Materials, University of Birmingham for the TEM images shown in this paper and continued valuable discussions. The crystal plasticity code employed here is an evolution of the code developed by Dr. C. Bronkhorst of Los Alamos National Laboratory and his colleagues and he is thanked for sharing this code with AWE.

(C) British Crown Owned Copyright 2019/AWE. Published with permission of the Controller of Her Britannic Majesty's Stationary Office.

\section{DATA AVAILABILITY}

The raw/processed data required to reproduce these findings cannot be shared at this time due to technical or time limitations. 


\section{REFERENCES}

[1] N.R. Barton, J.V. Bernier, R. Becker, A. Arsenlis, R. Cavallo, J. Marian, M. Rhee, H.S. Park, B.A. Remington, R.T. Olson, A multiscale strength model for extreme loading conditions, J. Appl. Phys, 109 (2011) 073501, https://doi.org/10.1063/1.3553718.

[2] K.G. Hoge, A.K. Mukherjee, The Temperature and Strain-rate Dependence of the Flow Stress of Tantalum, J. Mater. Sci., 12 (1977) 1666-1672, https://doi.org/10.1007/BF00542818.

[3] M. Kothari, L. Anand, Elasto-Viscoplastic Constitutive Equations for Polycrystalline Metals: Application to Tantalum, J. Mech. Phys. Sol., 46 (1998) 51-83, https://doi.org/10.1016/50225096(97)00037-9.

[4] P.J. Maudlin, J.F. Bingert, J.W. House, S.R. Chen, On the modeling of the Taylor cylinder impact test for orthotropic textured materials: experiments and simulations, Int. J. Plast., 15 (1999) 139166, https://doi.org/10.1016/S0749-6419(98)00058-8.

[5] L.E. Murr, M.A. Meyers, C.S. Niou, Y.J. Chen, S. Parru, C. Kennedy, Shock induced deformation twinning in tantalum, Acta Mater., 45 (1997) 157, https://doi.org/10.1016/S1359-6454(96)00145-0.

[6] B.W. Reed, J.R. Patterson, D.C. Swift, J.S. Stolken, R.W. Minich, M. Kumar, A unified approach for extracting strength information from nonsimple compression waves. Part II. Experiment and comparison with simulation, J. Appl. Phys., 110 (2011) 113506, https://doi.org/10.1063/1.3662173.

[7] A. Lawley, H.L. Gaigher, Deformation Structures in Zone-Melted Molybdenum, Phil. Mag., 10 (1964) 15-33, https://doi.org/10.1080/14786436408224204.

[8] S.R. Chen, G.T. Gray III, Consititutive Behavior of Tantalum and Tantalum-Tungsten Alloys, Metall. Mater. Trans. A, 27A (1996) 2994-3006, https://doi.org/10.1007/BF02663849.

[9] E. Schmid, W. Boas, Kristallplastizitat: Mit Besonderer Berucksichtigung der Metalle, Springer, 1935.

[10] J.F. Byron, Plastic deformation of tantalum single crystals. II. The orientation dependence of yield, J. Less-Common Metals, 14 (1968) 201-210, https://doi.org/10.1016/0022-5088(68)90115-X.

[11] J.F. Byron, D. Hull, Plastic deformation of tantalum single crystals. I. The surface morphology of yield, J. Less-Common Metals, 13 (1967) 71-84, https://doi.org/10.1016/0022-5088(67)90048-3.

[12] C.R. Weinberger, B.L. Boyce, C.C. Battaile, Slip Planes in BCC Transition Metals, Int. Mat. Rev., 58:5 (2013) 296-314, https://doi.org/10.1179/1743280412Y.0000000015.

[13] J.R. Asay, T. Ao, T.J. Vogler, J.-P. Davis, G.T. Gray III, Yield strength of tantalum for shockless compression to 18 GPa, J. Appl. Phys, 106 (2009) 073515, https://doi.org/10.1063/1.3226882.

[14] K.E. Duprey, R.J. Clifton, Pressure-shear response of thin tantalum foils, in: M.D. Furnish, L.C. Chhabildas, R.S. Hixson (Eds.) Shock Compression of Condensed Matter - 1999, Woodbury, NY, AIP Press, 2000, pp. 447-450.

[15] J.N. Florando, B.S. El-Dasher, C. Chen, D.C. Swift, N.R. Barton, J.M. McNaney, K.T. Ramesh, K.J. Hemker, M. Kumar, Effect of strain rate and dislocation density on the twinning behavior in tantalum, AIP Advances, 6 (2016) 045120, https://doi.org/10.1063/1.4948528.

[16] M.D. Furnish, D.H. Lassila, L.C. Chhabildas, D.J. Steinberg, in: S.C. Schmidt, W.C. Tao (Eds.) High Press. Sci. Techn., AIP, 1995, pp. $527-530$. 
[17] G.T. Gray III, N.K. Bourne, J.C.F. Millett, Shock response of tantalum: Lateral stress and shear strength through the front, J. Appl. Phys., 94 (2003) 6430, https://doi.org/10.1063/1.1620679.

[18] G.T. Gray III, K.S. Vecchio, Influence of peak pressure and temperature on the structure /property response of shock-loaded Ta and Ta-10W, Metall. Mater. Trans. A, 26A (1995) 2555-2563, https://doi.org/10.1007/BF02669413.

[19] D. Lassila, G.T. Gray III, Effects of shock prestrain on the dynamic mechanical behavior of tantalum, J. de Phys. IV, 01 (C3) (1991) C3_19-C13_26, 10.1051/jp4:1991303.

[20] J.C.F. Millett, G. Whiteman, N.T. Park, S. Case, N.K. Bourne, The Role of Cold Work on the Shock Response of Tantalum, J. Appl. Phys, 113 (2013) 233502, https://doi.org/10.1063/1.4810896.

[21] J.T. Lloyd, J.D. Clayton, R. Becker, D.L. McDowell, Simulation of Shock Wave Propagation in Single Crystal and Polycrystalline Aluminium, Int. J. Plast., 60 (2014) 118-144, https://doi.org/10.1016/j.ijplas.2014.04.012.

[22] T.E. Mitchell, W.A. Spitzig, Three-stage hardening in tantalum single crystals, Acta Metall., 13 (1965) 1169-1179, https://doi.org/10.1016/0001-6160(65)90054-4.

[23] A.J. Schwartz, W.E. King, G.H. Campbell, J.S. Stölken, D.H. Lassila, S. Sun, B.L. Adams, Orientation imaging microscopy Investigation of the Compression Deformation of a [011] Ta single crystal, J. Eng. Mat. Technol., 121 (1999) 178-181, https://doi.org/10.1115/1.2812364.

[24] P.J. Sherwood, F. Guiu, H.C. Kim, P.L. Pratt, Plastic Anisotropy of Tantalum, Niobium and Molybdenum, Canadian J. Phys., 45 (1967) 1075-1089, https://doi.org/10.1139/p67-079.

[25] W.A. Spitzig, T.E. Mitchell, Dislocation arrangements in tantalum single crystals deformed in tension at 373K, Acta Metall., 14 (1966) 1311-1323, https://doi.org/10.1016/0001-6160(66)90248-3.

[26] J.W. Christian, Some Surprising Features of the Plastic Deformation of Body Centred Cubic Metals and Alloys, Metall. Trans. A, 14A (1983) 1237-1256, https://doi.org/10.1007/BF02664806.

[27] D. Rittel, M.L. Silva, B. Poon, G. Ravichandran, Thermomechanical behaviour of single crystal tantalum in the static and dynamic regime, Mech. Mat., 41 (2009) 1323-1329, https://doi.org/10.1016/j.mechmat.2009.08.001.

[28] R. Kapoor, S. Nemat-Nasser, High-rate deformation of single crystal tantalum: temperature dependence and latent hardening, Scr. Mater., 40 (1999) 159-164.

[29] J.R. Asay, T.J. Vogler, T. Ao, J.L. Ding, Dynamic yielding of single crystal Ta at strain rates of $\sim 5 \mathrm{x}$ 105 /s, J. Appl. Phys., 109 (2011) 073507, https://doi.org/10.1063/1.3562178.

[30] J.L. Ding, J.R. Asay, Modeling of the dynamic inelasticity of tantalum single crystal under ramp wave loading, J. App. Phys, 109 (2011) 083505, https://doi.org/10.1063/1.3561376.

[31] J.N. Florando, N.R. Barton, B.S. El-Dasher, J.M. McNaney, M. Kumar, Analysis of Deformation Twinning in Tantalum Single Crystals under Shock Loading Conditions, J. Appl. Phys., 113 (2013) 083522, https://doi.org/10.1063/1.4792227.

[32] L.M. Hsuing, D.H. Lassila, Shock-induced deformation twinning and omega transformation in tantalum and tantalum-tungsten alloys, Acta Mater., 48 (2000) 4851-4865, https://doi.org/10.1016/S1359-6454(00)00287-1. 
[33] G. Whiteman, S. Case, J.C.F. Millett, Planar shock compression of single crystal tantalum from 6 - $23 \mathrm{GPa}$, Shock Compression of Condensed Matter - 2013, Institute of Physics, Journal of Physics: Conference Series, 2013, pp. 641-644.

[34] C.H. Lu, B.A. Remington, B.R. Maddox, B. Kad, H.S. Park, S.T. Prisbrey, M.A. Meyers, Laser Compression of Monocrystalline Tantalum, Acta Mater., 60 (2012) 6601-6620, https://doi.org/10.1016/j.actamat.2012.08.026.

[35] C.A. Bronkhorst, B.L. Hansen, E.K. Cerreta, J.F. Bingert, Modeling the microstructural evolution of metallic polycrystalline materials under localization conditions, J. Mech. Phys. Sol., 55 (2007) 2351-2383, https://doi.org/10.1016/j.jmps.2007.03.019.

[36] P. Gillis, K.G. Hoge, R.J. Wasley, Elastic Precursor Decay in Tantalum, J. Appl. Phys., 42 (1971) 2145, https://doi.org/10.1063/1.1660508.

[37] J.N. Johnson, O.E. Jones, T.E. Michaels, Dislocation Dynamics and Single Crystal Constitutive Relations: Shock Wave Propagation and Precursor Decay, J. Appl. Phys, 41 (1970) 2330, https://doi.org/10.1063/1.1659227.

[38] N.R. Barton, N. Winter, J.E. Reaugh, Defect Evolution and Pore Collapse in Crystalline Energetic Materials, Modelling Simul. Mater. Sci. Eng., 17 (2009) 035003, https://doi.org/10.1088/09650393/17/3/035003.

[39] D.L. Higgins, School of Metallurgy and Materials Science, University of Birmingham, Birmingham, 2017.

[40] B. Pang, School of Metallurgy and Materials Science, University of Birmingham, Birmingham, 2015.

[41] B. Pang, S. Case, I.P. Jones, J.C.F. Millett, G. Whiteman, Y.L. Chiu, C.A. Bronkhorst, The defect evolution in shock loaded tantalum single crystals, Acta Mat., 148 (2018) 482-491, https://doi.org/10.1016/j.actamat.2017.11.052.

[42] B. Pang, I. Jones, J.C.F. Millett, G. Whiteman, N.K. Bourne, Orientation dependence of shock induced dislocations in tantalum single crystals, J. Phys. Conf Series, 522 (2014) 012029, https://doi.org/10.1088/1742-6596/522/1/012029.

[43] B. Pang, I. Jones, J.C.F. Millett, G. Whiteman, N.K. Bourne, Y.L. Chiu, Radial stress release wave induced twinning in tantalum single crystals, Metall. Mater. Trans. A, (2014), https://doi.org/10.1007/s11661-014-2399-6.

[44] G. Whiteman, D.L. Higgins, B. Pang, J.C.F. Millett, Y.L. Chiu, I.P. Jones, Variations in hardness with position in one dimensionally recovered shock loaded metals, 12th Int. DYMAT Conf., European Physical Journal - Web of Conferences 183, Arcachon, France, 2018, pp. 02013, https://doi.org/10.1051/epjconf/201818302013.

[45] C.A. Bronkhorst, S.R. Kalidindi, L. Anand, Polycrystalline Plasticity and the Evolution of Crystallographic Texture in FCC Metals, Phil. Trans. R. Soc. Lond., 341 (1992) 443-477, https://doi.org/10.1098/rsta.1992.0111.

[46] S.R. Kalidindi, C.A. Bronkhorst, L. Anand, Crystallographic texture evolution in bulk deformation processing of FCC metals, J. Mech. Phys. Sol., 40 (1992) 537-569, https://doi.org/10.1016/00225096(92)80003-9. 
[47] R.A. Austin, D.L. McDowell, A Dislocation-based Constitutive Model for Viscoplastic Deformation of FCC Metals at Very High Strain-rates, Int. J. Plast., 27 (2011) 1-24,

https://doi.org/10.1016/j.ijplas.2010.03.002.

[48] F. Roters, P. Eisenlohr, L. Hantcherli, D.D. Tjahjanto, T.R. Bieler, D. Raabe, Overview of Constitutive Laws, Kinematics, Homogenization and Multiscale Methods in Crystal Plasticity Finite Element Modelling: Theory, Experiments, Applications, Acta Mat., 58 (2010) 1152-1211, https://doi.org/10.1016/j.actamat.2009.10.058.

[49] J.W. Christian, S. Mahajan, Deformation Twinning, Prog. Mater. Sci., 39 (1995) 1-157, https://doi.org/10.1016/0079-6425(94)00007-7.

[50] L. Stainer, A.M. Cuitino, M. Ortiz, A Micromechanical Model of Hardening, Rate Sensitivity and Thermal Softening in BCC Single Crystals, J. Mech.Phys. Solids, 50 (2002) 1511-1545.

[51] Smallman, R.E. (1985), Modern Physical Metallurgy, Butterworth and Co (publishers) Ltd. 